\title{
Under what kind of parametric fluctuations is spatiotemporal regularity the most robust?
}

\author{
Manish Dev Shrimali* \\ Department of Physics, Dayanand College, Ajmer, 305 001, India \\ Swarup Poria ${ }^{\dagger}$ \\ Department of Mathematics, Midnapore College, \\ Midnapore, 721 101, West Bengal, India \\ Sudeshna Sinha \\ The Institute of Mathematical Sciences, \\ Taramani, Chennai 600 113, India
}

\begin{abstract}
It was observed that the spatiotemporal chaos in lattices of coupled chaotic maps was suppressed to a spatiotemporal fixed point when some fraction of the regular coupling connections were replaced by random links. Here we investigate the effects of different kinds of parametric fluctuations on the robustness of this spatiotemporal fixed point regime. In particular we study the spatiotemporal dynamics of the network with noisy interaction parameters, namely fluctuating fraction of random links and fluctuating coupling strengths. We consider three types of fluctuations: (i) noisy in time, but homogeneous in space; (ii) noisy in space, but fixed in time; (iii) noisy in both space and time. We find that the effect of different kinds of parameteric noise on the dynamics is quite distinct: quenched spatial fluctuations are the most detrimental to spatiotemporal regularity; spatiotemporal fluctuations yield phenomena similar to that observed when parameters are held constant at the mean-value; and interestingly, spatiotemporal regularity is most robust under spatially uniform temporal fluctuations, which in fact yields a larger fixed point range than that obtained under constant mean-value parameters.
\end{abstract}

\footnotetext{
* e-mail: m.shrimali@gmail.com

$\dagger$ e-mail: swarup_p@yahoo.com

$\ddagger$ e-mail: sudeshna@imsc.res.in
} 


\section{INTRODUCTION}

One of the important prototypes of extended complex systems are nonlinear dynamical systems with spatially distributed degrees of freedom, or alternately spatial systems composed of large numbers of low dimensional nonlinear systems. The basic ingredients of such systems are: (i) creation of local chaos or local instability by a low dimensional mechanism and (ii) spatial transmission of energy and information by coupling connections of varying strengths and underlying topologies.

The Coupled Map Lattice (CML) is such a model, capturing the essential features of the nonlinear dynamics of extended systems [3]. A very well-studied coupling form in CMLs is nearest neighbour coupling. However some degree of randomness in spatial coupling can be closer to physical reality than strict nearest neighbour scenarios. In fact many systems of biological, technological and physical significance are better described by randomising some fraction of the regular links [1, 2]. Here we focus on a ring of coupled chaotic maps whose coupling connections are dynamically rewired to random sites with probability $p$, namely at any instance of time, with probability $p$ a regular link is switched to a random one [4].

It has recently been found that such random coupling yields a spatiotemporal fixed point in a network of chaotic maps [5]. That is, the strongly unstable fixed point of the local chaotic map is stabilized under increasing randomness in the coupling connections. Thus interestingly, the inherent chaos present in the individual local units is suppressed by dynamically switched random links, giving rise to a global spatiotemporal fixed point attractor.

In this paper we study the effect of parametric fluctuations on the synchronization properties of such networks. We consider different types of noise in the parameters: (i) spatial (ii) temporal and (ii) both spatial and temporal. Keeping the local dynamics always fully chaotic, we focus on parameteric noise in the interaction parameters between the nodes in the network. In particular, we consider fluctuations in the fraction of random links $p$ in the system, and fluctuations in the coupling strength of the different links. That is, we study perturbations in both the geometry of the network connections, as reflected in noisy $p$, as well as in the strength of the links. 


\section{MODEL}

We consider a network of $\mathrm{N}$ coupled logistic maps. The sites are denoted by integers $i=1, \ldots, N$, where $N$ is the size of the lattice. On each site is defined a continuous state variable denoted by $x_{n}(i)$, which corresponds to the physical variable of interest.

The evolution of this lattice, under interactions with the nearest neighbours, is given by

$$
x_{n+1}(i)=(1-\epsilon) f\left(x_{n}(i)\right)+\frac{\epsilon}{2}\left\{x_{n}(i+1)+x_{n}(i-1)\right\}
$$

The strength of coupling is given by $\epsilon$. The local on-site map is chosen to be the fully

chaotic logistic map: $f(x)=\alpha x(1-x)$ with $\alpha=4$, as this map has widespread relevance as a prototype of low dimensional chaos.

We consider the above system with its coupling connections rewired randomly with probability $p$. Namely, at every update we will connect a site with probability $p$ to randomly chosen sites, and with probability $(1-p)$ to nearest neighbours, as in Eqn. 1. That is, at every instant a fraction $p$ of randomly chosen nearest neighbour links are replaced by random links. The case of $p=0$ corresponds to the usual nearest neighbour interaction, while $p=1$, corresponds to completely random coupling. This type of connectivity has been observed in a range of natural and human-engineered systems [2].

In this work, we will focus on the effect of fluctuations in the interaction parameters, i.e. noisy coupling strength $\epsilon$, and fraction of random links $p[\underline{6}]$. Now, one can have four distinct scenarios (denoting the relevant parameter as $A$ ):

(i) $A_{n}(i) \equiv A_{0}$

Here the parameter is constant. We will denote this case of zero fluctuations by $C$.

(ii) $A_{n}(i)=A_{0} \pm \delta A \eta^{i} \equiv A(i)$

Here $\delta A$ is the strength of the fluctuation in the parameter around mean value $A_{0}$ and $\eta^{i}$ is a zero-mean random number. So here the parameters are random in space but remain frozen in time, i.e. the parameters are spatially fluctuating but temporally invariant. In this case then, we have quenched disorder or a basic inhomogeneity of the network links. We will denote this case of spatial parametric fluctuations by $S$.

(iii) $A_{n}(i)=A_{0} \pm \delta A \eta_{n} \equiv A_{n}$.

Again $\delta A$ is the strength of the fluctuation in the parameter around mean value $A_{0}$ and $\eta_{n}$ is a zero-mean random number. So the fluctuation is a function of time but is site 
independent, i.e. the noise in the parameter is synchronous for all the elements, namely parameter $A$ is spatially uniform, though random in time. This kind of a situation may arise when the system is quite uniform intrinsically, but is subject to a common perturbation, for instance from a common environmental influence, like say fluctuations in the ambient temperature. We will denote this case of temporal parametric fluctuations by $T$.

(iv) $A_{n}(i)=A_{0} \pm \delta A \eta_{n}^{i}$

Here the fluctuations are a function of both time and space. Such a scenario describes a situation where the system is both inhomogeneous in space and noisy in time. We will denote this case of spatiotemporal parametric fluctations by ST.

Here we consider $\eta$ to be uniformly distributed in the interval $[0,1]$. We have simulated the system, for all the above cases, with fluctuations in coupling strength (i.e. $A \equiv \epsilon$ ) and in the fraction of random links (i.e. $A \equiv p$ ). The initial conditions of the individual elements were randomly chosen in the interval $[0,1]$, and sufficient transients were removed before looking at the spatiotemporal profile of the network.

\section{ROBUSTNESS OF THE SPATIOTEMPORAL FIXED POINT UNDER PARAMET- RIC FLUCTUATIONS}

Fig. 1 displays the bifurcation diagrams of the system under different types of fluctuations in the fraction of random links $p$, around a mean value of $p_{0}$. It is evident that the spatiotemporal fixed point is quite robust under parametric fluctuations in general, as the range of the spatiotemporal fixed point in coupling parameter space does not reduce much under noisy $p$. It can also clearly be seen that the bifurcation profile of the system under spatiotemporal fluctuations in $p$ (Fig. 1d) is very similar to the system under constant $p=p_{0}$ (Fig. 1a). Further we observe that quenched spatial fluctuations reduces the range of stability of the spatiotemporal fixed point most significantly (Fig. 1b). On the other hand temporal fluctuations in $p$ does not degrade the stability of the fixed point regime, and is most conducive to spatiotemporal regularity. In fact, interestingly the fixed point range obtained under temporal prametric fluctuations (Fig. 1c) is larger than that obtained from the constant case (Fig. 1a). Qualitatively similar bifurcation diagrams with respect to $p$ were obtained under fluctuations in the coupling strength of the different links.

In order to quantify the above observation we calculate the average deviation of the 
system from a synchronized state, denoted by $Z$, and defined as:

$$
Z=<<\left(x_{n}(i)-\bar{x}\right)^{2}>>
$$

where $\bar{x}$ is the mean value of $x$. The averages $\langle<\ldots>>$ are over all sites $i(i=1, \ldots N)$ and over long times $n$.

Figs. 2-3 show error $Z$ with respect to coupling strength and the fraction of random links $p$, under parametric fluctuations in $p$ and $\epsilon$ respectively. It is clearly evident from both figures that temporal parametric fluctuations $(T)$ gives zero error for the largest range. Spatiotemporal parametric fluctuations $(S T)$ and the case where the parameter is kept constant at the mean value $(C)$ give completely similar trends. As observed earlier, the case of spatial paramteric fluctuations $(S)$ gives the smallest range, namely the spatiotemporal fixed point is least robust under quenched disorder.

We also calculate the critical value of coupling strength after which the spatiotemporal fixed point gains stability, denoted by $\epsilon_{c}$, and the critical fraction of random links after which the spatiotemporal fixed point is stable, denoted by $p_{c}$. The fixed point range in coupling parameter space is then $\left(1-\epsilon_{c}\right)$, and in the space of $p$ it is $\left(1-p_{c}\right)$.

Figs. 4-5, shows the range of the spatiotemporal fixed point in $\epsilon$ and $p$ space, for varying strengths of parameteric fluctuations. It is clear that the effect of spatiotemporal fluctuations is quite indistinguishable from the mean field case (namely the case of $p_{0}$ and $\epsilon_{0}$, with $\delta p$ and $\delta \epsilon$ equal to zero). It is also evident that quenched disorder reduces the stable range the most, while the fixed point range is the largest for spatially uniform temporal fluctuations. In fact remarkably, for temporal fluctuations, the fixed point range for large fluctuation strengths is larger than that obtained for zero fluctuations. These results re-inforce the conclusions drawn from the calculations of the error function $Z$.

Lastly note that similar trends are also observed when the parametric fluctuations are periodic, not random. For instance consider the case where the fraction of random links $p$ is distributed in a period 2 cycle $: p_{1}, p_{2}$. For the case of quenched spatial periodic fluctuations (denoting the value of $p$ at site $i$ as $p(i)$ ) we have: $p_{1}(1), p_{2}(2), p_{1}(3), p_{2}(4) \ldots$ for all time. For temporal periodic fluctuations we have $p(i)=p_{1}$ for all sites $i$ at time $n$, followed by $p(i)=p_{2}$ for all sites at time $n+1$, back to $p(i)=p_{1}$ for all sites at time $n+2$ etc... For spatiotemporal periodic fluctuations we have the parameters varying as a 2-cycle in space and time, namely $p_{1}(1), p_{2}(2), p_{1}(3), p_{2}(4) \ldots$ at time $n$ followed by $p_{2}(1), p_{1}(2), p_{2}(3), p_{1}(4) \ldots$ at 

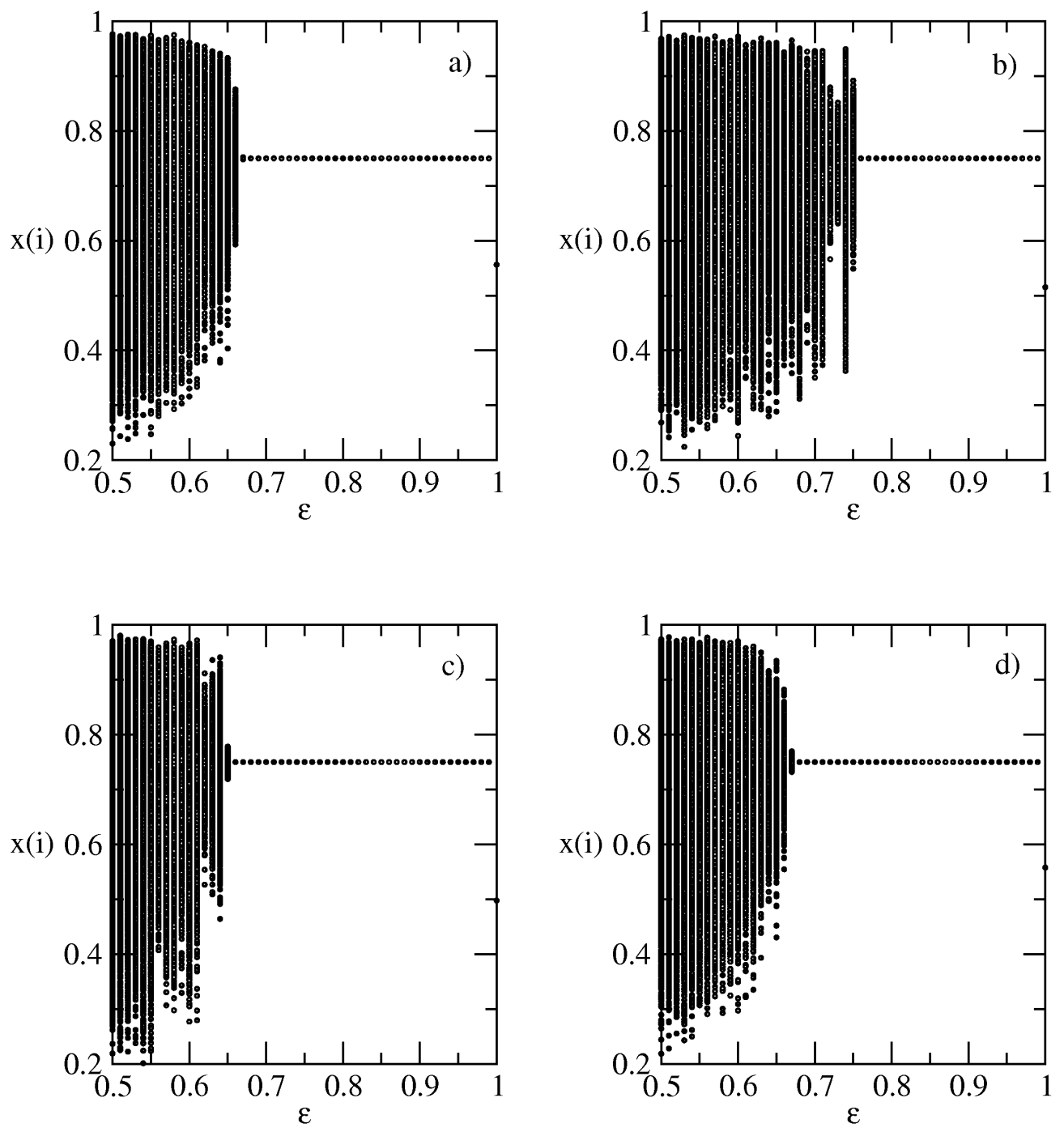

FIG. 1: State of the system with respect to coupling strength $\epsilon$, under different types of fluctuations in $p$ : (a) constant $p=0.5$ (b) spatial fluctuations (c) temporal fluctuations and (d) spatiotemporal fluctuations. Here strength of fluctuations $\delta p=0.5$ in cases $(\mathrm{b})-(\mathrm{d})$, around a mean value of $p_{0}=0.5$.

time $n+1$ etc... For all these cases one obtains the same qualitative behavior as the random fluctuation cases, namely quenched spatial periodic fluctuations are the most detrimental to spatiotemporal regularity; space-time periodic fluctuations yield phenomena similar to that with constant mean-values $p_{0}=\left(p_{1}+p_{2}\right) / 2$; and spatiotemporal regularity is most robust under spatially uniform periodic fluctuations. 


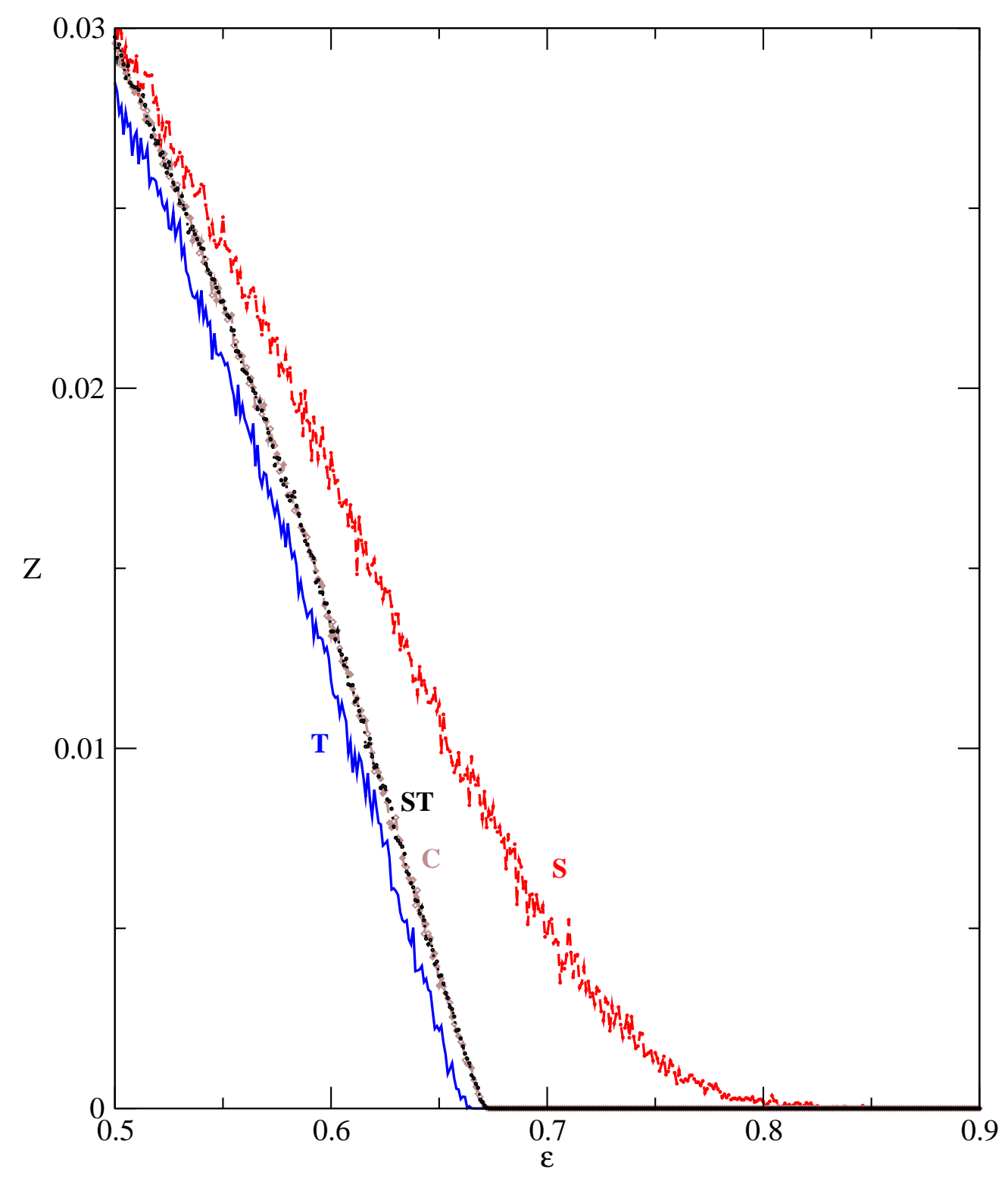

FIG. 2: Average synchronization error $Z$, as a function of coupling strength, for four different cases: (i) $p$ constant at the mean value $p_{0}=0.5$ denoted by $C$; (ii) spatial fluctuations in $p$ denoted by $S$; (iii) temporal fluctuations in $p$ denoted by $T$, and (iv) spatiotemporal fluctuations in $p$ denoted by $S T$. Here the strength of fluctuations $\delta p=0.5$ in cases (ii)-(iv), namely $p$ is distributed uniformly in the range $[0: 1]$. Observe that $T$ gives (almost) zero error for the largest range, $S T$ and $C$ give similar trends, while $S$ gives the smallest range, namely the least robustness for the spatiotemporal fixed point. 


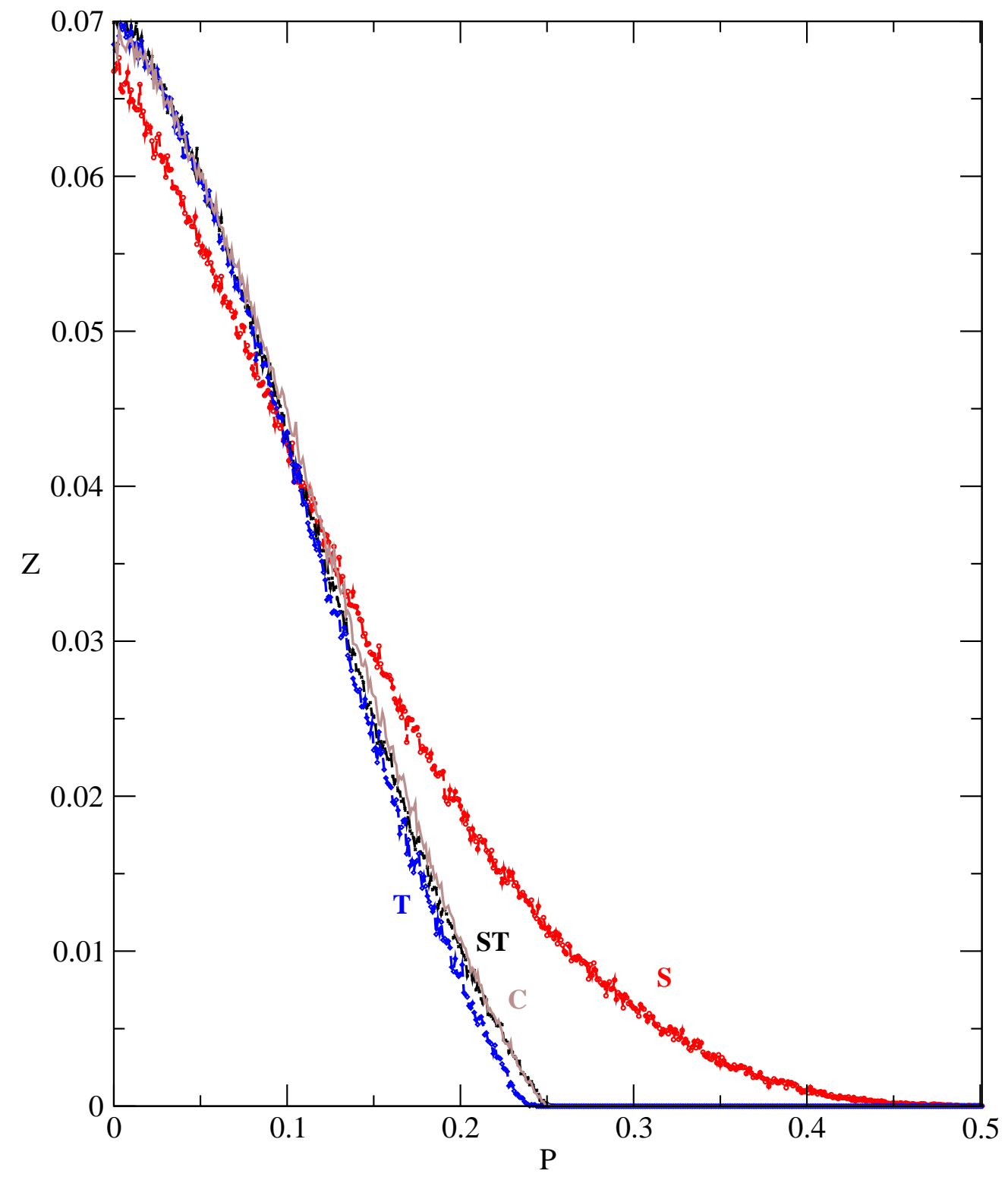

FIG. 3: Average deviation of the system from the spatiotemporal fixed point, $Z$, as a function of the fraction of random links $p$, for four different cases: (i) $\epsilon$ constant at the mean value $\epsilon_{0}=0.8$ denoted by $C$; (ii) spatial fluctuations in $\epsilon$ denoted by $S$; (iii) temporal fluctuations in $\epsilon$ denoted by $T$, and (iv) spatiotemporal fluctuations in $\epsilon$ denoted by $S T$. Here the strength of fluctuations $\delta \epsilon=0.2$ in cases (ii)-(iv), namely $\epsilon$ is distributed uniformly in the range [0.6:1]. Observe that $T$ gives (almost) zero error for the largest range, $S T$ and $C$ give similar trends, while $S$ gives the smallest range, namely the least robustness for the spatiotemporal fixed point. 


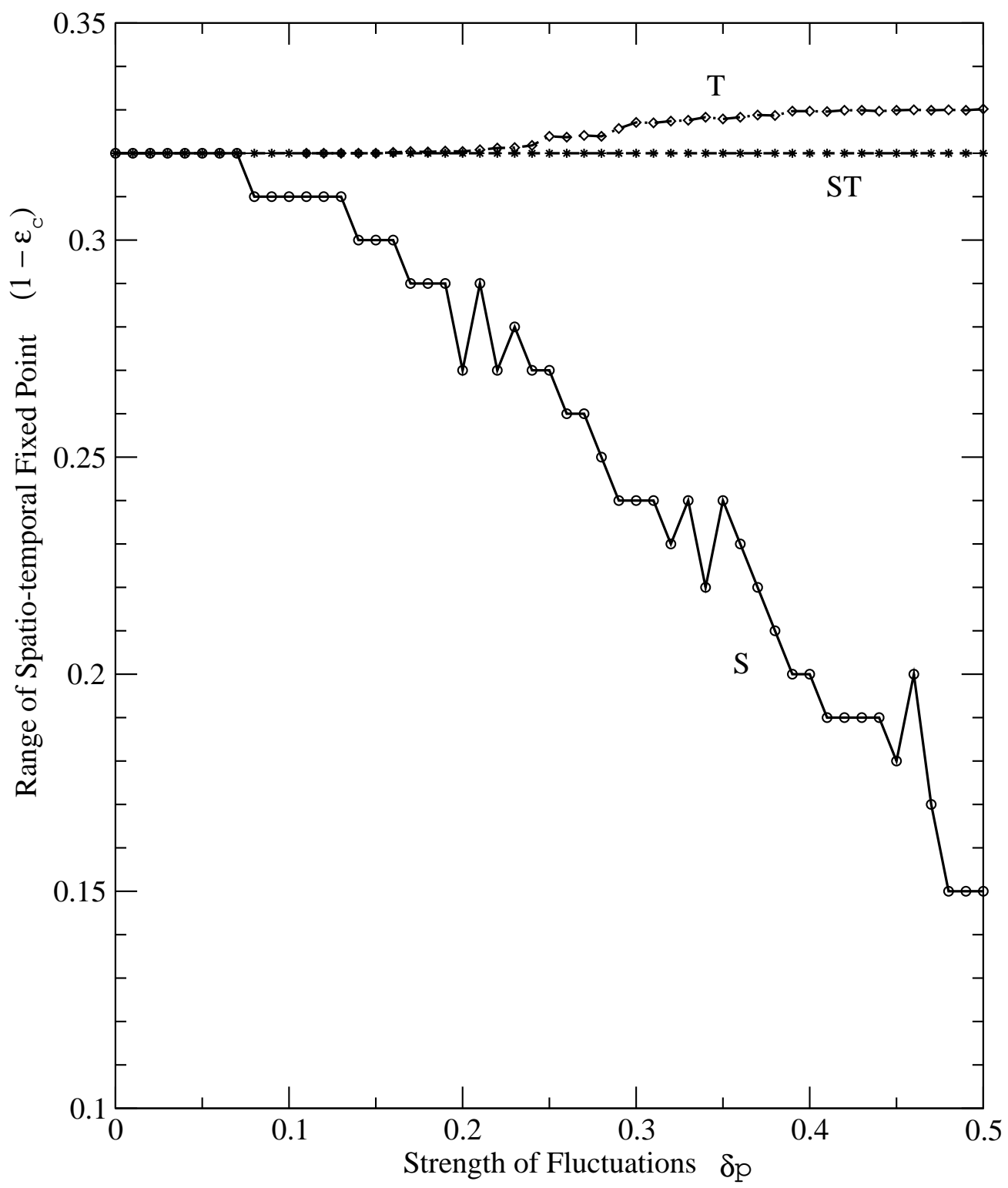

FIG. 4: Range of the spatiotemporal fixed point $\left(1-\epsilon_{c}\right)$, where $\epsilon_{c}$ is the critical value of coupling strength after which the spatiotemporal fixed point gains stability, as a function of the parametric fluctuation strength $\delta p$. Note that fluctuation strength of $\delta p$ implies that the fraction of random links is distributed uniformly in the range $\left[p_{0}-\delta p: p_{0}+\delta p\right]$, with the mean $p_{0}=0.5$. Observe that temporal fluctuations of reasonbly large strength $\delta p$, actually increases the fixed point range, vis-a-vis the case of zero fluctuations $\delta p=0$ (i.e. the constant $p_{0}$ case). 


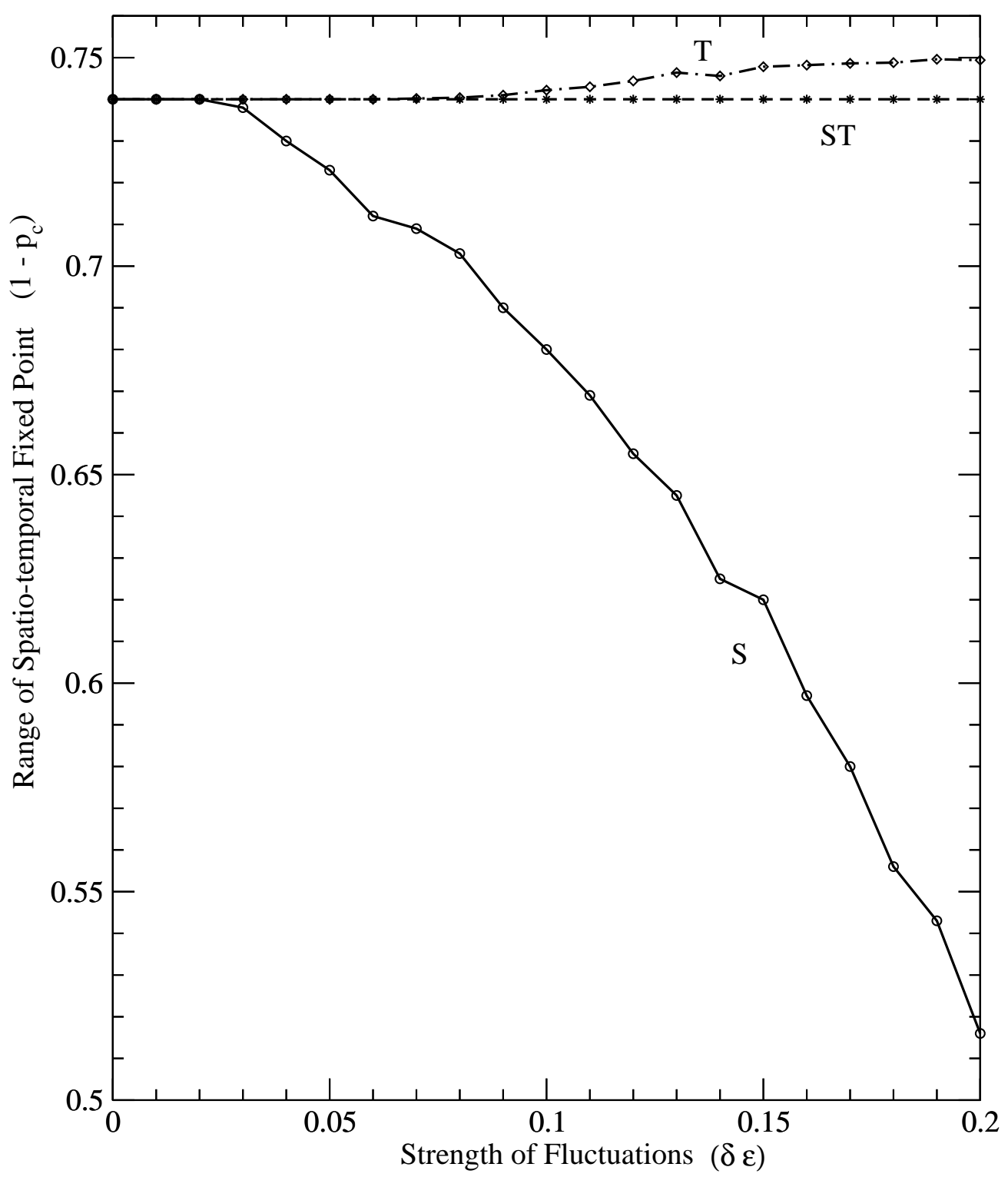

FIG. 5: Range of the spatiotemporal fixed point $\left(1-p_{c}\right)$, where $p_{c}$ is the critical value of $p$ after which the spatiotemporal fixed point gains stability, as a function of the parametric fluctuation strength $\delta \epsilon$. Note that fluctuation strength of $\delta \epsilon$ implies that coupling $\epsilon$ is distributed uniformly in the range $\left[\epsilon_{0}-\delta \epsilon: \epsilon_{0}+\delta \epsilon\right]$, with the mean $\epsilon_{0}=0.8$. Observe that temporal fluctuations of reasonbly large strength $\delta \epsilon$, actually increases the fixed point range, vis-a-vis the case of zero fluctuations $\delta \epsilon=0$ (i.e. the constant $\epsilon_{0}$ case). 


\section{CONCLUSIONS}

It was observed that lattices of coupled chaotic maps, with coupling connections dynamically rewired to random sites with probability $p>0$, gave rise to a window of spatiotemporal fixed points in coupling parameter space. Here we investigate the effects of different kinds of parametric fluctuations on the robustness of this spatiotemporal fixed point regime. In particular we study the spatiotemporal dynamics of the network with fluctuating rewiring probabilities and coupling strengths, with the fluctuations being (a) noisy in time, homogeneous in space, as applicable for intrinsically homogenoeus systems under common environmental noise; (ii) noisy in space, and fixed in time, namely quenched disorder; and (iii) noisy in both space and time..

We find that static spatial inhomogeniety, namely quenched disorder, degrades spatiotemporal regularity most significantly. Spatiotemporal fluctuations yield dynamical properties almost identical to networks with the parameters held constant at the mean values. Interestingly, spatiotemporal regularity is most robust under spatially uniform temporal fluctuations. Such space-invariant temporal parametric noise actually yields a regular range that is larger than that obtained for systems with the parameters held constant at mean-value.

So the effect of different kinds of parameteric noise on spatiotemporal regularity is quite distinct: quenched spatial fluctuations are the most detrimental to spatiotemporal regularity; spatiotemporal fluctuations yield phenomena similar to that with constant mean-values; and spatiotemporal regularity is most robust under spatially uniform temporal fluctuations.

[1] R. Albert and A.-L. Barabasi, Rev. of Mod. Phys. 74, 47 (2002), A.-L. Barabasi, 'Linked: The New Science of Networks' (Massachusetts: Persus Publishing, 2002); T. Gross and B. Blasius, J. of Royal Soc. - Interface 5259 (2008).

[2] D. J. Watts and S. H. Strogatz, Nature, 393440 (1998).

[3] K. Kaneko, Theory and Applications of Coupled Map Lattices, Wiley, New York (1993)

[4] P. M. Gade and C.-K. Hu, Phys. Rev. E 626409 (2000); Z. Gao, B. Hu and G. Hu, Phys. Rev. E 65016209 (2001); M. Barahona and L. Pecora, Phys. Rev. Lett., 89, 054101 (2002); J. Jost and M. P. Joy, Phys. Rev. E 65 016201(2002); M. G. Cosenza and K. Tucci, Phys. Rev. E, bf 650326223 (2002); P. R. A. Campos, V. M. de Oliveira and F. G. Brady Moreia, Phys. 
Rev. E 67026104 (2003); P.M. Gade and S. Sinha, Int. J. of Bif. and Chaos, 162767 (2006); M.P.K. Jampa et al, Phys. Rev. E, 75026215 (2007); F. Radicchi and H. Meyer-Ortmanns, Phys. Rev. E 74026203 (2006); T. Gross, C.J.D'Lima and B. Blasius, Phys. Rev. Letts. 96 208701 (2006); S. Rajesh et al, Phys. Rev. E, 75011906 (2007); S. Poria, M. D. Shrimali, and S. Sinha, submitted.

[5] S. Sinha, Phys. Rev. E 66, 016209 (2002).

[6] There are some studies on uncoupled and globally coupled chaotic maps under the influence of fluctuations in the local nonlinearity parameter. See for instance: S. Sinha, Phys. Rev. Lett. 69, 3306 (1992); G. Perez, H.A. Cerdeira, Phys. Rev. E 49, 15 (1994); S. Sinha, Phys. Lett. A 245, 393 (1998). 